\title{
Grain growth in sintering: a discrete element model on large packings
}

\author{
Brayan Paredes-Goyes ${ }^{\mathrm{a}}$, David Jauffres ${ }^{\mathrm{a}}$, Jean-Michel Missiaen ${ }^{\mathrm{a}}$, \\ Christophe L. Martin ${ }^{\mathrm{a}}$ \\ ${ }^{a}$ Univ. Grenoble Alpes, CNRS, Grenoble INP, SIMaP, F-38000 Grenoble, France
}

\begin{abstract}
Sintering is a high temperature process used for ceramic or metallic powder consolidation that consists of concurrent densification and grain growth. This work presents a coupled solid-state sintering and grain growth model capable of studying large packings of particles within the Discrete Element Method (DEM) framework. The approach uses a refinement for large particle size ratios of previously established contact laws to model shrinkage. In addition, mass transfer between neighboring particles is implemented to model grain growth by surface diffusion and grain-boundary migration. The model assumptions are valid for initial and intermediate stage sintering. The model is validated on a two-particle system by comparing neck and particle size evolutions with those obtained by phase-field and meshedbased methods. Simulations on large packings (up to 400000 particles) with particle size distributions originating from experiments are performed. The results of these simulations using physical data from the literature are compared to experimental data with good accordance of the key features of the microstructure evolution (densification kinetics, grain size-density trajectory, evolution of the mean grain size and of the size distribution). The simulations show that even at an early stage of sintering, hardly detectable grain growth actually affects the sintering kinetics to a non-negligible extent and that the realism of DEM simulations of sintering is improved when grain growth is considered. Taking advantage of the possibility to simulate large packings, the model elucidates the influence of the initial particle size distribution on the grain growth kinetics.
\end{abstract}

Keywords: sintering, grain growth, grain boundary migration, grain size distribution, discrete element method

Preprint submitted to Acta Materialia 


\section{Introduction}

The sintering of metallic or ceramic powders is a high temperature process occurring below the melting temperature. It leads to the consolidation of the powder by growing necks between particles while generally decreasing the porosity of the packing [1]. The fundamental driving force for sintering is the reduction of the interfacial energy of the system, which, in addition to the reduction of the free surface, can result in grain growth $[2,3]$. For relatively dense powder compact (green density 0.5-0.6), grain growth is mostly observed in the intermediate and final stages of sintering (typically for relative density $D>0.8$ ) and is driven by grain boundary migration, leading to the coarsening of larger grains at the expense of the smaller ones and to an increase of the average grain size $\bar{G}$. Grain growth kinetics is classically described by a power law of the type $\bar{G}^{n}-\bar{G}_{0}^{n} \propto t$ with $\bar{G}_{0}$ the initial grain size. Under the assumption of grain growth by grain-boundary (GB) migration the theoretical value of the exponent $n$ is 2 for a dense body [4] while for a body with closed porosity the growth is slower due to the pinning of the grain boundaries by closed pores leading theoretically to $n=3$ for volume diffusion and $n=4$ for surface diffusion [5]. Experimental data on sintering confirms an exponential type law but with $\mathrm{n}$ generally close to $3[3,6]$. Lange and Kellet [2] have described grain growth during sintering of porous compacts with a broad particle size distribution by inter-particle mass transport followed by GB migration. For a wide variety of ceramics and metals, surface diffusion is the most relevant mechanisms of inter-particle mass transport $[7,8]$. Lange and Kellet scenario is in line with experimental observation: a linear grain size evolution with fractional porosity at lower densities, followed by a non-linear evolution in the late stages of sintering [3]. On the other hand, Bernard-Granger et al. have shown that a wide range of experimental data on alumina is correctly described by a theoretical relationship between $\bar{G}$ and the relative density $D$ derived under the assumption of grain growth by GB migration $\left(1 / \bar{G}^{2}-1 / \bar{G}_{0}^{2} \propto D\right)$ in solid-state sintering, which tends to demonstrate that GB migration is the dominant mechanism $[9,10]$.

The control of grain growth during sintering is an important topic as coarse microstructures are generally detrimental for material performance, in particular for mechanical properties. When the grain size distribution maintains the self-similarity predicted theoretically [4] the grain growth is referred to as normal but under specific conditions a few large grains can exhibit very fast growth, giving rise to so-called abnormal grain growth, 
characterized by a significant broadening of the grain size distribution. An explanation often provided is that large GB mobility appears locally because of a non-uniform distribution of impurities or secondary phase [8]. Indeed, the presence of secondary phase at a GB can influence positively or negatively its mobility, a phenomenon that can also be advantageously used to limit grain growth $[3,11,12]$. Recently, numerical modeling of sintering coupled with grain growth have been proposed through finite difference method [13], Monte Carlo (MC) model [14, 15, 16, 17, 18], phase field approach $[19,20,21,22,23,24]$, finite element or meshed-based methods $[25,26]$, Discrete Element Method (DEM) [27] or a combination of methods $[28,29]$. Due to the complexity of the representation of the shape and of the physics of sintering, these approaches are, with the exception of DEM, generally computationally limited to a few particles, rarely a few hundreds, and often in 2D. Still, simulations with relatively large number of particles have been performed using a Monte Carlo model [14, 15, 16, 17, 18]. The advantage of such simulations are their capability to describe the evolution of realistic microstructures with all the necessary kinetic processes that come with solid-state sintering. This type of simulation is able to provide useful information on grain size evolution with density. However, in the MC method, model parameters and time (Monte Carlo steps) may not have a clear physical meaning. In addition, MC models are limited to free sintering [30].

Grain growth at the later stage of sintering has also been simulated with phase field simulations on a relatively large 3D system by Rehn et al. [31], starting with an initial configuration of small isolated pores at triple lines and quadruple junctions. Recently, approaches have been proposed using initial random packings of spherical particles [32,33], where starting from an initial configuration typically obtained by discrete simulations, the authors perform 3D phase field simulations with the main diffusion mechanisms simulated (surface, grain-boundary, and bulk diffusion). The effect of rigidbody motion of individual particles may also be included [33]. In the latter study, the authors were able to run simulations with about 3000 particles and up to a final relative density of around 0.8. Although, the number of particles is already a great improvement, it comes at the cost of massive CPU parallelization (120 CPUs) and may not be sufficient as many grains disappear with coarsening at large relative densities (typically above 0.70 relative density).

Thus, there is still a need for further improvement for numerical sim- 
ulations that operate at the particle scale to obtain valuable information on the microstructure evolution. The evolution of size distribution, which would necessitate large number of particles for statistics, or the influence of large defects have not been studied for example. Indeed, it would be beneficial to have access to simulations that provide such information with typically 10 times the number of particles and only a fraction of the CPU cost, while retaining the main physical ingredients that govern sintering and grain growth. For example, starting with several tens of thousands of particles would allow for a statistically representative size distribution even at large densities where the number of particles may have decreased down to less than a thousand.

In this context, following the initial work of Parhami and Mc Meeking [34], Martin et al. [27] have used DEM [35] to model sintering of tens of thousands of particles. Nevertheless, most sintering investigations based on DEM [34, 36, 37, 38, 39, 40,41] do not take into account grain growth and coarsening of particles. To our best knowledge, only one DEM study [27] includes a crude model of grain growth that does not consider realistic driving forces at the scale of individual particles. Still, grain growth should be included in large-scale simulations as coarsening and sintering are intimately linked and grain growth affects sintering kinetics [2]. Even in the early stage of sintering, the realism of DEM simulations can benefit from the addition of a physically based grain growth model. Also, DEM provides a natural mean to introduce realistic initial packing with size distribution $[41,42,43,44]$. For packings with size distribution, sintering contact models that handle particles of different sizes are necessary. Whereas most DEM simulations deal with equal size particles $[27,36,37,45]$ or use an equivalent radius by analogy with elastic and plastic contact theories [37, 39, 41, 42], it is necessary to introduce more realistic models for unequal size particles with large size ratios. Pan et al. [13] proposed such a description based on numerical simulations at the scale of individual particles, but to our best knowledge no DEM simulation has yet introduced this type of model.

The aim of this work is thus to propose a discrete model of the sintering of a packing of particles under equilibrated sintering forces coupled with a grain growth model for particles of different sizes. The model, applied at the particle scale, should be sufficiently realistic to agree with state-ofthe-art phase field simulations that operate at much smaller length scales, while taking advantage of the fully discontinuous framework of DEM to simulate large packings that can be statistically useful for further analy- 
sis. The model is limited to initial and intermediate stage sintering. In the model description section we first briefly delineate the DEM methodology and the model focusing on the description of the sintering contact laws that include: the normal and tangential contact forces, the evolution of the contact size and equilibrium contact size for two particles unequal in size. The proposed grain growth model is then detailed with the necessary conditions for triggering each mechanism (surface diffusion and GB migration). The model results for two particles are analyzed and validated against phase field simulation for each stage of the sintering process. In the last section, the sintering of 40,000 and 400,000 alumina particle packings are simulated for various GB mobility and initial particle size distribution. The results are compared to experiments and discussed in light of existing laws for grain growth kinetics and mean grain size - density trajectory.

\section{Model description}

The model is developed in the DEM code dp3D, dedicated to materials science and already used for sintering studies over the last 15 years [27, 46, 47, 48]. Here we briefly describe its general scheme. Each particle is a single crystallographic grain and is considered as a sphere which upon densification can indent its neighbors. Note that unlike in the description proposed by Lange and Kellet [2] there is no distinction between particles and grain and thus GB migration is bound to be an inter-particle/grain mass transport. The main geometrical parameters defining two particles in contact are given in Fig. 1, where $r_{s}$ and $r_{l}$ are the radius of the smaller and larger particles, respectively, $a$ is the contact radius and $h$ is their mutual indentation. Unlike the classical DEM approach, the radius of particles can evolve depending on matter diffusion driven by curvature gradient. Particles interact through their contacts that transmit forces. Rotations are not allowed here as they are rapidly opposed by resisting moments when contact size becomes finite. Contact forces are summed for each particle and the total force is used to compute explicitly the acceleration, velocity and the new position of each particle using Newton's second law with a velocity Verlet algorithm.

Contact detection is a critical stage for the computational efficiency of large DEM simulations. This is especially true here since grain growth implies the coexistence of particles of very different sizes along the simulation as large particles will grow at the expense of smaller ones. Standard detection schemes for nearly monomodal packings such as Verlet list together 

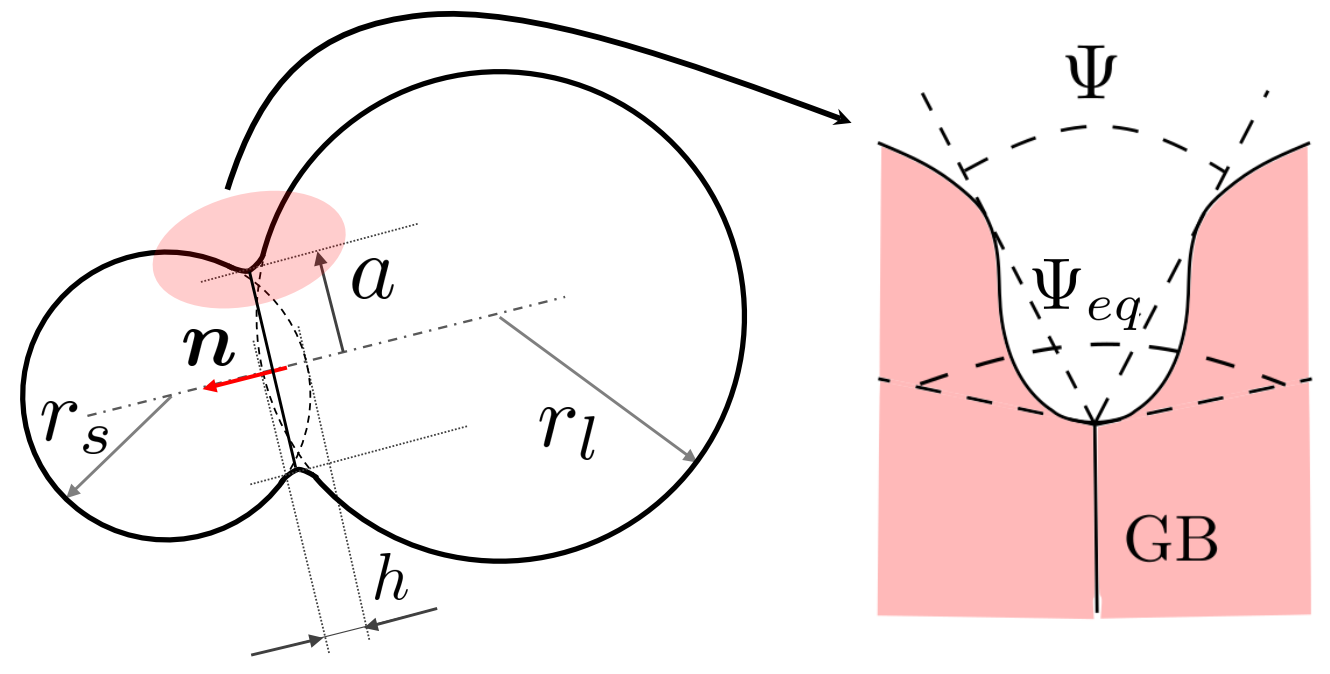

Figure 1: Geometrical parameters for a large particle (radius $r_{l}$ ) sintering with a small one (radius $r_{s}$ ). $h, a$ and $\boldsymbol{n}$ are the geometric indentation, the contact radius and the normal vector, respectively. $\Psi$ is the contact angle and $\Psi_{e q}$ is the equilibrium dihedral angle at the grain boundary GB.

with the Linked-Cell method are insufficient in that case. A fast multilevel algorithm as proposed by Ogarko and Luding [49] was implemented to resolve effectively this issue.

Free sintering is modeled here within a periodic box in all three directions. The stress tensor $\Sigma_{p q}$ is calculated from Love's formulation using the $p^{t h}$ component of the total contact force vector $\boldsymbol{F}$ and the $q^{\text {th }}$ component of the branch vector that connects the two particle centers [50]:

$$
\Sigma_{p q}=\frac{1}{V} \sum_{\text {contacts }} F_{p}\left(r_{s}+r_{l}-h\right) n_{q}
$$

where the summation is made on all contacts with normal vector $\mathbf{n}$ (Fig. 1 ), and $V$ is the volume of the periodic box. The macroscopic strain-rates are imposed to the simulation box, such that the principal components of the macroscopic stress tensor $\Sigma_{p q}$ tends to zero at each time-step. Note that the same scheme can be used for stress-assisted sintering.

\subsection{Contact laws for sintering}

The normal force between two sintering particles in contact is derived from the models of Bouvard and McMeeking [45] and of Pan et al. [13]. The 
Bouvard and McMeeking model applies to pairs of particles of identical size whereas Pan et al. fitted their results for two particles of different sizes with a similar expression as Bouvard and McMeeking. These expressions can be rearranged to derive the normal force $N$ between two particles with radii $r_{s}$ and $r_{l}$ :

$$
N=-\frac{\pi a^{4}}{\left(1+\frac{r_{s}}{r_{l}}\right) \beta \Delta_{G B}} \frac{\mathrm{d} h}{\mathrm{~d} t}+\frac{\alpha}{\beta} \pi r_{l} \gamma_{S}
$$

which introduces the surface energy $\gamma_{S}$ and a diffusion-related term:

$$
\Delta_{G B}=\frac{\Omega}{k_{b} T} D_{G B} \delta_{G B}
$$

where $D_{G B}=D_{0 G B} \exp \frac{-Q_{G B}}{R T}$ is the diffusion coefficient along the grain boundary with activation energy $Q_{G B}$ at temperature $T, \delta_{G B}$ the grainboundary thickness, $k_{b}$ the Boltzmann constant and $\Omega$ the atomic volume. The $\alpha$ and $\beta$ parameters depend on the ratio of the grain-boundary diffusion to surface diffusion $\xi=\delta_{G B} D_{G B} / \delta_{S} D_{S}$ [45]. Here, for a given temperature, grain-boundary and surface diffusion coefficients $D_{G B}$ and $D_{S}$ were chosen and $(\alpha ; \beta)$ were set in accordance with $\xi$ parameter (Table 1 and [27]). Eq. (2) introduces a viscous component (repulsive or attractive) that counteracts the relative approach of the two particles while the second term is always attractive and represents the force responsible for shrinkage. Another method for accounting for unequal size particles in Eq. (2), in analogy with elasticity, is to use the equivalent radius $\frac{r_{s} r_{l}}{r_{s}+r_{l}}[27,37,51]$. The two methods depart only markedly for large size ratios, for which Eq. (2) better captures the Pan et al. [13] finite difference results, with larger values of both the viscous and tensile components. Eq. (2) introduces the contact radius $a$ to the power 4 in the viscous term, thus accounting for the slower kinetics of sintering as $a$ increases. Our DEM model introduces a contact radius evolution equation. The Coble model [52], valid for equal-sized particles writes:

$$
a^{2}=2 r h
$$

2 For two particles of different sizes, Pan et al. [13] fitted their finite difference simulation results to obtain a generalized equation:

$$
a^{2}=\kappa\left[0.5\left(1+\frac{r_{s}}{r_{l}}\right)\right]^{\zeta} r_{l} h
$$

where $\kappa=2.4$ and $\zeta=1.5$ are fitted empirical values. Note that in the original Pan's equations (Eqs. (2) and (5)), the initial radii are considered 
whereas in our DEM model, we use the current radii. The proposed model reproduces correctly the original results from Pan et al. [13] (see section S1 of the Supplementary Information (SI)).

As the contact grows, the sum of the grain boundary and surface energies may reach a local minimum from which any perturbation of the contact shape increases the total energy. This equilibrium state is obtained when the contact angle $\Psi$ reaches the equilibrium dihedral angle $\Psi_{e q}$. The calculation of the corresponding equilibrium contact radius $a_{e q}$ is based on geometric considerations to obtain a relation between the contact angle $\Psi$ and the contact radius $a$, whatever mechanism is at play for its growth. This results in a set of nonlinear equations (see section S2 of the SI) that can be numerically resolved and fitted linearly reasonably well for a wide range of contact angles and particle size ratios leading to:

$$
a_{e q}=\frac{\Psi_{e q}}{\hat{\Psi}} \frac{r_{s}}{1+\frac{r_{s}}{r_{l}}}
$$

where $\hat{\Psi}=92.937^{\circ}$ is a fitted constant. This is a generalization of the work of Lange [2], which assumed a simplest contact geometry strictly defined by the intersection of two spheres. Finally, when $a=a_{e q}$ the equilibrium configuration is reached and the tensile shrinkage term in Eq. (2) is set to zero so that any additional growth of the contact requires a compressive force. Additionally to normal interactions (Eq. (2)), tangential viscous interactions are also introduced as detailed in [37], with a dimensionless viscous parameter $\eta=0.01$.

\subsection{Grain growth}

The evolution of the radius of a particle is calculated by considering the exchange of volume at each contact, with the volume flux always from the smaller to the larger particle. The sum of volume fluxes for each particle leads to an updated radius. When the volume of a given particle decreases below a critical value $\left(C \times \frac{4}{3} \pi r_{m, 0}^{3}\right)$ (with $r_{m, 0}$ the initial mean radius), the particle is simply removed from the box and its volume is equally distributed to all remaining particles. We checked that results are not affected by the value of $C$, providing $C \leq 10^{-3}$.

The values of the current contact radius (Eq. (5)) and contact equilibrium size (Eq. (6)) are used to activate specific grain growth mechanisms. When activated, the equation for the volume variation of a large particle $l$ 
in contact with a small one $s$ writes:

$$
\frac{\mathrm{d} V_{l, s}}{\mathrm{~d} t}=4 \pi r_{l}^{2} \frac{\mathrm{d} r_{l}}{\mathrm{~d} t}=\sum_{i} J_{i} A_{i} \Omega
$$

where the volume exchanged for a given contact $\mathrm{d} V_{l, s}$ is due to different mechanisms of mass transport (Surface diffusion $S$ or Grain-Boundary Migration $G B M)$, each one represented by a flux cross-section area $A_{i}$ and by an atomic flux density $J_{i}(i=S, G B M)$ :

$$
J_{i}=-\frac{D_{i}}{k_{b} T} \nabla P_{i}
$$

GB migration and surface diffusion are considered here as they are recognized as the two mass transport mechanisms contributing to grain growth as discussed in the introduction. Both mass fluxes can be represented by a generic form, where $D_{i}$ is the diffusion coefficient of the mechanism, and $\nabla P_{i}$ the Laplace pressure gradient that causes mass transfer. The volume variation of the smaller particle $s$ in contact with the larger one $l$ is $\frac{\mathrm{d} V_{s, l}}{\mathrm{~d} t}=-\frac{\mathrm{d} V_{l, s}}{\mathrm{~d} t}$, thus ensuring volume conservation.

Both grain growth mechanisms are based on the curvature gradient as the driving force. The curvature difference is related with the chemical potential [53], which is proportional to the local Laplace pressure gradient. Denoting $\gamma_{S}$ and $\gamma_{G B}$ the surface and grain boundary energies, for two spherical particles the Kelvin equation leads to a Laplace pressure difference of $2 \gamma_{S}\left(\frac{1}{r_{l}}-\frac{1}{r_{s}}\right)$ and $2 \gamma_{G B}\left(\frac{1}{r_{l}}-\frac{1}{r_{s}}\right)$ for surface diffusion and GB migration, respectively $[2,54]$. Although reasonable for surface diffusion, this expression is a simplification of the real configuration for GB migration. First, it is considered that grain boundary interfaces are dominant when grain boundary migration is active and, as proposed in mean field theories of grain growth $[4,55]$, a mean Laplace pressure difference at particle scale is used.

The activation criteria for these mechanisms are based on equilibrium considerations. For surface diffusion, matter from the smaller particle has to flow to the neck before migrating to the larger particle. Therefore, to allow mass transfer between particles, the local neck curvature cannot be concave. This configuration occurs when two particles in contact reach the equilibrium configuration [2], i.e. the contact angle $\Psi$ reaches the equilibrium dihedral angle $\Psi_{e q}$. Hence, grain growth by surface diffusion is activated 
once the condition $a \geq a_{e q}$ is fulfilled. (Fig. 2 stage 2). At this stage, as stated above, the shrinkage term in Eq. (2) is set to zero (Fig. 2 stage 2).

For GB migration, the growth is activated when thermodynamically favorable conditions are met, i.e. when the grain boundary area does not increase during GB migration [2]. Thus, GB migration is activated in our model when the contact radius is equal or greater than the radius of the smallest particle (Fig. 2 stage 3).

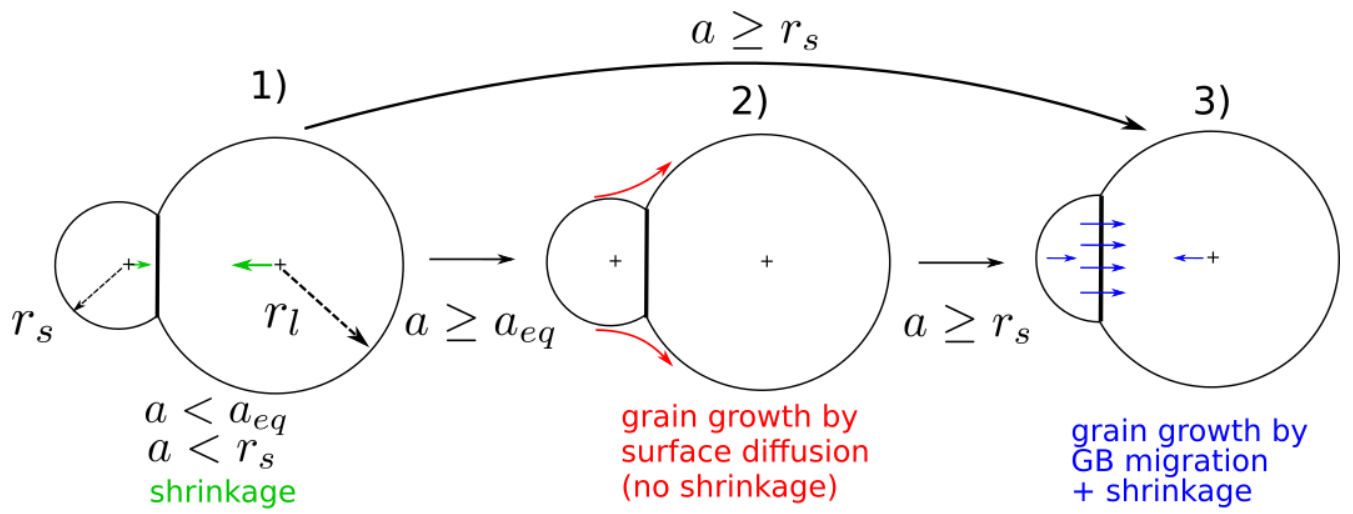

Figure 2: Possible mechanisms activated for sintering and grain growth. 1) Standard sintering with shrinkage without grain growth when the neck is sufficiently small. 2) Surface diffusion without shrinkage when the neck radius is larger than or equal to the equilibrium neck radius (Eq. (6)). 3) GB migration with shrinkage when the neck radius is larger than or equal to the smallest particle radius. Note that mechanism 1) may lead directly to 3 ).

The pressure gradient calculation in Eq. (8) necessitates the definition of a proper distance to write the local Laplace pressure gradient. For surface diffusion, we choose the center to center distance, $\left(r_{s}+r_{l}-h\right)$, considering that it represents a suitable average distance for the flux of matter. For GB migration, instead of using the transverse grain boundary diffusion coefficient $D_{G B M}$ as the input parameter we introduce the more convenient and often used grain-boundary mobility, $M_{G B}=\frac{D_{G B M} \Omega}{k_{b} T \delta_{G B}}$. Hence, the considered diffusion distance is implicitly the grain boundary thickness $\delta_{G B}$. At the the macroscopic scale, the grain-boundary mobility depends on the porosity $[56,57]$ and the grain-boundary misorientation $[58,59]$. In our model, we consider the intrinsic grain-boundary mobility [60], that depends only on temperature via an Arrhenius law [56]. Porosity is indirectly taken into account by the local configuration of contacts. Additionally, the grain- 
boundary and surface energies are considered constant. The exchange area for surface diffusion is a circular ring of radius $a$ and thickness the surface diffusion layer $\delta_{S}$. For GB migration, it is the entire area of the neck, that is considered as circular.

The DEM implicit assumption of indented spherical particles is no more fulfilled for the small particle at the later stage of grain growth [13, 22]. The DEM geometrical sphere simplification leads to a very small contact area in the last instants of grain growth which unrealistically slows down mass transport. Thus, we assume this area to be constant (with $a^{*}$ the related neck radius) for the computation of matter fluxes and contact forces from the beginning of GB migration. With these simplifying assumptions, the following contributions for the fluxes of matter by Surface diffusion $(i=S)$ and Grain-Boundary migration $(i=G B M)$ write:

$$
\left(\frac{\mathrm{d} V_{l, s}}{\mathrm{~d} t}\right)_{S}=-2 \frac{D_{S}}{k_{b} T} \gamma_{S} \Omega \frac{\frac{1}{r_{l}}-\frac{1}{r_{s}}}{r_{l}+r_{s}-\delta}\left[\pi\left(a+\delta_{S}\right)^{2}-\pi a^{2}\right]
$$

$$
\left(\frac{\mathrm{d} V_{l, s}}{\mathrm{~d} t}\right)_{G B M}=-2 M_{G B} \gamma_{G B}\left(\frac{1}{r_{l}}-\frac{1}{r_{s}}\right)\left[\pi a^{* 2}\right]
$$

Both coefficients $M_{G B}$ and $D_{S}$ introduce temperature dependence through Arrhenius law with pre-exponential factors $M_{0 G B}$ and $D_{0 S}$, and activation energies $Q_{G B M}$ and $Q_{S}$, respectively.

To sum up, Eqs (9) and (10) are applied at each time step for each contact when appropriate conditions are met by $a$. Three scenarios are possible as sketched in Fig. 2 depending on the values of the contact radius. The shrinkage force is reactivated in stage 3 . This reactivation is required to avoid unrealistic losses of contacts when particle size ratio becomes too large. In addition, for the sake of simplicity and considering that once GB migration is activated it is the dominant mechanism [9, 10], matter transport by surface diffusion is not active for a contact in stage 3 . Note also that stage 3 may arise either from stage 1 or 2 . The model thus creates a coupling between grain growth and sintering kinetics, which will be studied in the next sections.

\section{Results and discussion}

The grain growth mechanisms considered above are valid for a wide variety of ceramics and metals. To illustrate the accuracy of the model, we choose to apply it to alumina, as literature provides extensive material 


\begin{tabular}{|c|c|c|c|}
\hline$\delta_{G B} D_{0 G B}\left(\mathrm{~m}^{3} / \mathrm{s}\right)$ & $1.3 \times 10^{-8}[64]$ & $Q_{G B}(\mathrm{~kJ} / \mathrm{mol})$ & $475[65]$ \\
\hline$D_{0 S}^{*}\left(\mathrm{~m}^{2} / \mathrm{s}\right)$ & $0.09[62]$ & $Q_{S}(\mathrm{~kJ} / \mathrm{mol})$ & $313.8[62]$ \\
\hline $\begin{array}{c}M_{0 G B}^{*} \\
\left(\mathrm{~m}^{3} /(\mathrm{N} . \mathrm{s})\right)\end{array}$ & $0.02[56]$ & $Q_{G B M}(\mathrm{~kJ} / \mathrm{mol})$ & $443[56]$ \\
\hline$\Psi_{e q}\left(^{\circ}\right)$ & $138[61]$ & $\Omega\left(\mathrm{m}^{3}\right)$ & $2.11 \times 10^{-29}[62]$ \\
\hline$\gamma_{S}\left(\mathrm{~J} / \mathrm{m}^{2}\right)$ & $0.905[62]$ & $\gamma_{G B}\left(\mathrm{~J} / \mathrm{m}^{2}\right)$ & $2 \gamma_{S} \cos \left(\Psi_{e q} / 2\right)$ \\
\hline$\alpha^{1}$ & 2.46 or $2.48[45]^{1}$ & $\beta$ & $4[45]$ \\
\hline$r_{m, 0}(\mu \mathrm{m})$ & $0.2[66]$ & $\sigma_{0}$ & $0.23[66]$ \\
\hline
\end{tabular}

Table 1: Parameters used in the simulations for alumina. Diffusion coefficients and mobility are functions of the temperature $T$ with Arrhenius dependence of the form $\exp \left(\frac{-Q}{R T}\right)$. Simulations are conducted at $T=1350^{\circ} \mathrm{C}$.

data (see Table 1). No fitting parameter is used in this comparison. A wide variation of $D_{S}$ is reported in the literature as discussed by Tsoga and Nikolopoulos [61]. We chose the data of Robertson and Chang [62] (powder from Morganite inc.) as they were obtained for the largest temperature range $\left(1100-1720^{\circ} \mathrm{C}\right)$. This leads to a ratio between grain-boundary and surface diffusion $\xi=0.001$. Likewise $M_{G B}$ has a wide range that depends strongly on porosity [56] and on the presence of dopants [11,63]. To our knowledge, experimental GB mobility data for porous alumina (relative density $<0.95$ ) is available in literature only for temperatures above $1600^{\circ} \mathrm{C}$ $[11,12]$. We chose the GB mobility from [56] measured for the largest temperature range $\left(1325-2020^{\circ} \mathrm{C}\right)$. As this data is for dense alumina and the GB mobility $M_{G B}$ is one of the most relevant parameters affecting grain growth, we will study the influence of lower values of $M_{G B}$. All parameters used in the simulations are shown in Table 1.

\subsection{Sintering of two particles}

First, we compare our results with other approaches on a simple configuration made of two unequally sized particles. Kumar et al. [22] have tackled this problem through numerical simulations by representing thermodynamic quantities in the system by phase fields and minimizing its total free energy (bulk free energy, surface and grain boundary energy). Using a surface mesh and the Surface Evolver program, Wakai et al. [25] also provide numerical solutions with a rather different method. Note that the 
initial ratio of the two particles is different in the two studies $\left(\frac{r_{s, 0}}{r_{l, 0}}=0.5\right.$ and 0.75 ) and that Wakai et al. consider sintering by evaporation-condensation instead of coupled GB/surface diffusion as in Kumar's study and the present one. Fig. 3 compares the evolution of the contact radius and of the radius of the smaller particle (both normalized by their maximum values) from the present study to those of these prior works. Two simulations with different values of the initial size ratio at $1350^{\circ} \mathrm{C}$ for alumina were carried out. Three stages corresponding to the three possible mechanisms modeled (Fig. 2) are clearly visible on the simulations: initial neck growth without changes in radii followed by a second stage characterized by a slow decrease of the small particle radius at a nearly constant neck size and a third stage with both fast decrease in neck size and small particle radius.

The evolution of the contact size and the small particle size (Fig. 3) are in correct qualitative accordance with both Kumar et al. and Wakai et al. albeit an earlier onset of grain growth for their simulations. Also the transitions between each stages are more gradual in their simulations as in our model different mechanisms cannot occur simultaneously (e.g. grain growth by surface diffusion and GB migration). In addition, in our model an underestimation of the mass transport by surface diffusion is possible due to the distance chosen $\left(r_{s}+r_{l}-h\right)$ for the gradient in Eq. (8) as this approximation is reasonable if we assume a linear gradient. In all practical situations where the gradient is not linear, the gradient will be steeper.

In short, it can be concluded that our simplistic two-sphere scheme correctly reproduce the scenario predicted by both the phase field approach of Kumar et al. [22] and the surface evolver approach of Wakai et al. [25], but with significantly less computational effort. This is both mandatory, as we are aiming for several tens of thousands of particles in DEM, and encouraging as it means that DEM simulations will not compromise too much on accuracy compared to more elaborate methods.

The influence of the two main material parameters of the grain growth model, namely surface diffusivity and grain-boundary mobility is assessed. Fig. 4a indicates that, as expected, higher values of both parameters reduce the time for disappearance of the smallest particle. We checked that the values are in the same order of magnitude than that of Pan et al. [13]. Fig. 4a also clearly shows the necessity to include both mechanisms in a coarsening model at the length scale of particles. If only surface diffusion (first decay slope) is considered, it would take an unrealistically long time to remove the whole mass. Despite the rapid action of GB migration (second 


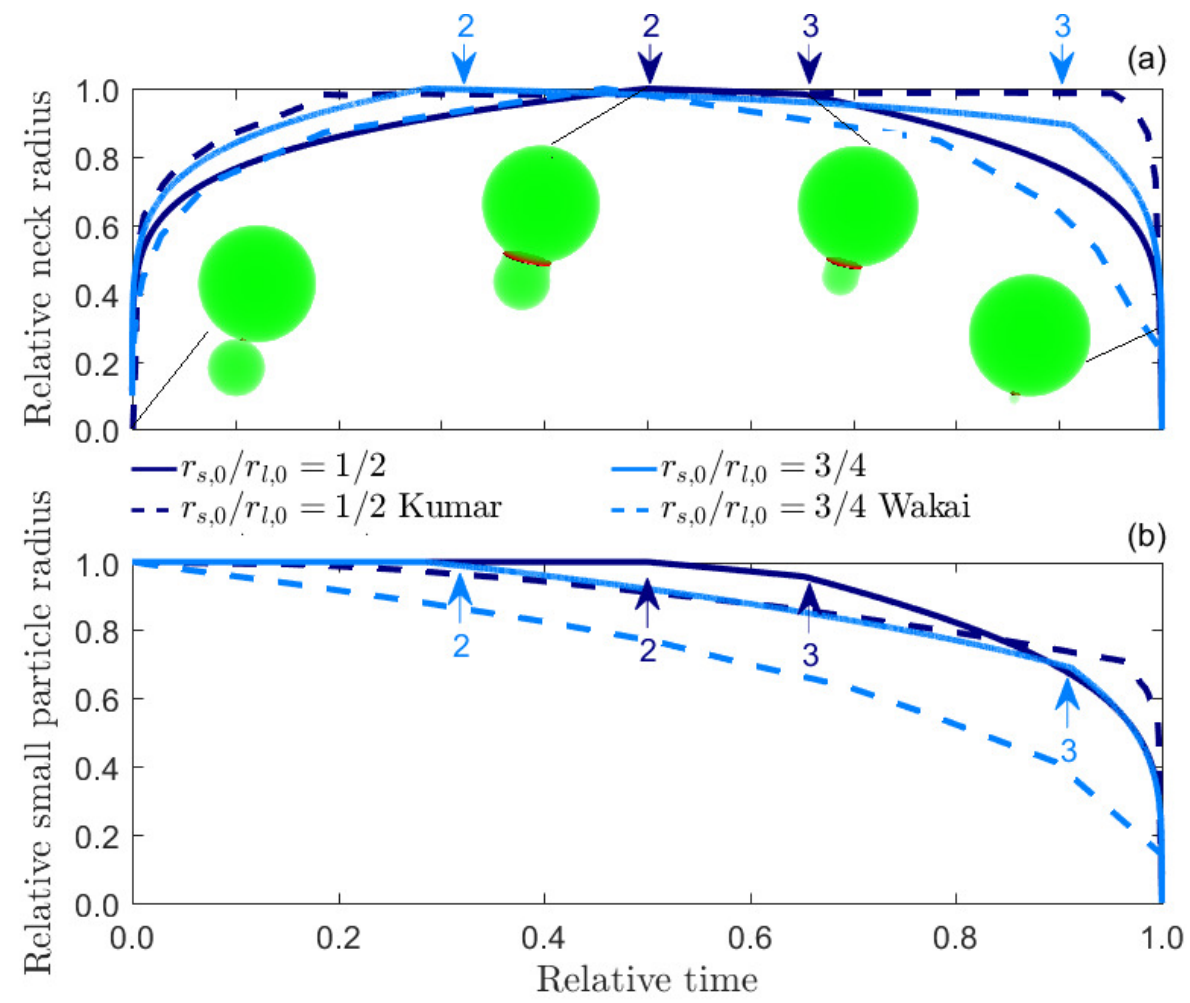

Figure 3: Evolution of the normalized contact radius $\frac{a}{\max (a)}$ (a) and of the normalized smaller particle radius $\frac{r_{s}}{\max \left(r_{s}\right)}$ (b) for two values of the initial size ratio $r_{s, 0} / r_{l, 0}$. Comparison with results from Kumar et al. [22] and Wakai et al. [25]. The numbers and arrows show the beginning of second (surface diffusion) and third (grain boundary migration) stages (Fig. 2). Time is normalized by the total time of disappearance of the smaller particle. Snapshots of the configuration of the pair of particles and the neck are given at various stages: initial, stage 2 and 3 and on the way to the final disappearance of the smallest particle. The images have been generated from particles positions and radii, and the computed neck size (Eq. (5)). The geometry used to represent the necks is two inverse tori tangent to each particle, which degenerate to a cylinder having the radius of the small particle in the last stage (see section $\mathrm{S} 2$ of the $\mathrm{SI}$ ).

decay slope), surface diffusion is required to reach the geometric starting conditions, i.e. the small particle size reaches the neck size.

Realistic initial green packings should feature particles with a wide range of size ratios. Fig. 4b displays the disappearance dynamics of the smallest particle for different initial size ratios. As expected from examining the gradient terms in Eqs. (9) and (10), the vanishing time is considerably shorter for smaller ratios, with two orders of magnitude, between the vanishing 

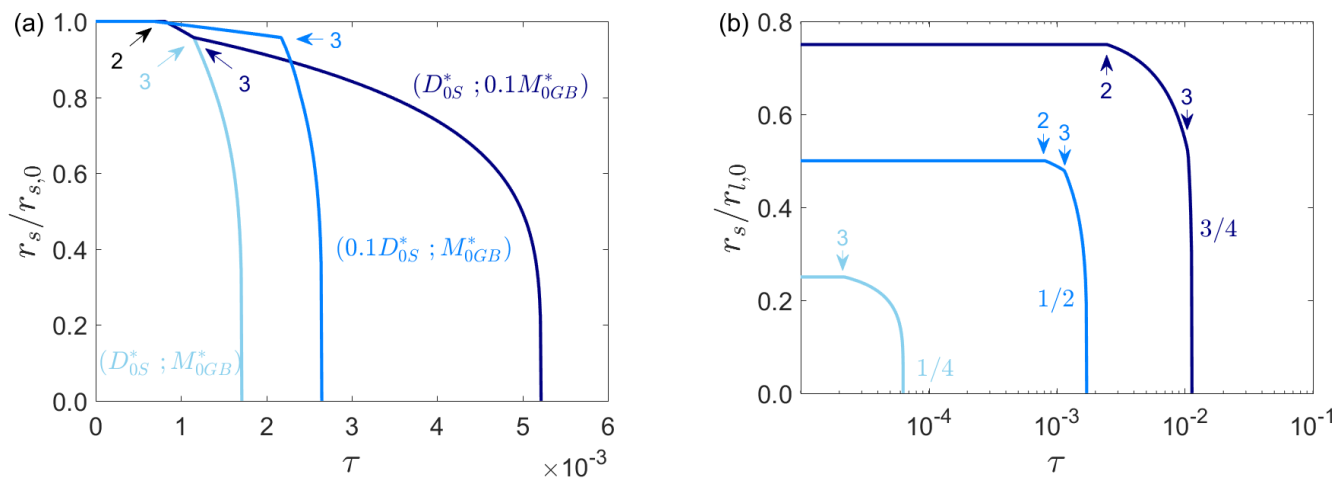

Figure 4: Evolution of the smaller particle radius with normalized time $\tau=\frac{r_{l, 0}^{4} k_{b} T}{\gamma_{S} D_{G B} \delta_{G B} \Omega}$ [13]. a) For different pair values of surface diffusivity $\left(D_{S}\right)$ and grain-boundary mobility $\left(M_{G B}\right)$ defined by the values in Table 1 . The radius of the smaller particle is normalized by its initial value $\left(r_{s 0}\right)$. b) For different initial size ratios between the smaller and larger particles. The radius of the smaller particle is normalized by the initial radius of the larger particle $\left(r_{l 0}\right)$. The numbers show the beginning of stage 2 (surface diffusion) and 3 (GB migration) ( see Fig. 2).

times of ratios $\frac{1}{4}$ and $\frac{3}{4}$. Surface diffusion, if activated, is the limiting stage for grain growth due to its duration. Interestingly, for ratio $\frac{1}{4}$ stage 2 is absent. This is because this configuration already exhibits, after sintering (stage 1), the geometrical conditions to immediately start grain growth by GB migration.

\subsection{Sintering of large packings of particles}

Packings made of 40,000 and 400,000 particles were used. The preparation procedure of the initial green packings is detailed in section S3 of the SI.

\subsubsection{Comparison to Nettleship experimental data}

In order to evaluate the newly formulated model at the scale of a large packing, we chose to focus on the only study on alumina that carefully reports the particle size distribution during sintering (see section S3 of the SI for a description of the lognormal $\left(\mu_{0}, \sigma_{0}^{2}\right)$ size distribution adopted here) [66]. Indeed, we observed that the initial particle size distribution significantly affects the simulation results, hence the need to have access to this information for a proper comparison. The simulation parameters are reasonable values from the literature (Table 1) and the initial particle size dis- 
tribution and green density match the experiments of Nettleship et al. The comparison is carried out in terms of grain size - relative density trajectory (Fig. 5a) and evolution of the lognormal law parameter $\sigma$ (Fig. 5b). Three different values of GB mobility were tested. The simulations were stopped either when they reached $D=0.95$ or when they experienced very rapid grain growth that was incompatible with numerical stability within reasonable computational times. Simulations show that GB mobility strongly affects the results and that the nominal value $M_{0 G B}^{*}$ taken from literature measurements on dense alumina is too high to reproduce the grain growth trajectory of Nettleship sintering experiment. A more appropriate value might lie between $0.25 M_{0 G B}^{*}$ and $0.5 M_{0 G B}^{*}$, and it is probable that this value evolves with density during the course of sintering [8]. Fig. 5b shows that the self-similarity (i.e. $\sigma=$ constant) observed in Nettleship experiments up to $D \approx 0.9$ is not correctly reproduced by simulations but the widening of the size distribution during the whole sintering is in reasonable agreement. At the onset of grain growth, before particles begin to disappear, a strict self-similarity is not expected in the simulations as mass transfer between particles increases the size of large particles and decreases the size of small particles, thus broadening the size distribution. The first part of simulations with a moderate increase of $\sigma$ can however be interpreted as a normal grain growth, in contrast to the faster increase of $\sigma$ observed for $M_{0 G B}^{*}$ that is typical of abnormal grain growth i.e. a fast increase in size of only a few particles. Such behavior is also observed for experimental data, but only above $D=0.95$. It is worth noting that while local variations of GB mobility (due to inhomogeneous distribution of impurities, crystallographic orientations, etc...) are generally claimed to be responsible for abnormal grain growth [8]; here, we observe that these, while surely exacerbating the phenomena, do not appear to be mandatory. Last, 40k and 400k packings exhibit very similar outputs indicating that 40k particles are sufficient to obtain representative results with periodic boundary conditions in this system.

Various visual representations of the microstructure evolution during a sintering simulation are provided in Fig. 6. It is interesting to note that at $D=0.85$ grain growth is clearly present visually while it is barely noticeable on the mean grain size value $\left(\frac{\bar{G}-\bar{G}_{0}}{G_{0}} \approx 1 \%\right)$. In other words, it is not required to observe a significant increase in mean grain size to have significant grain growth that might influence the microstructure and sintering kinetics. Another remarkable point is a global microstructure coarsening. 

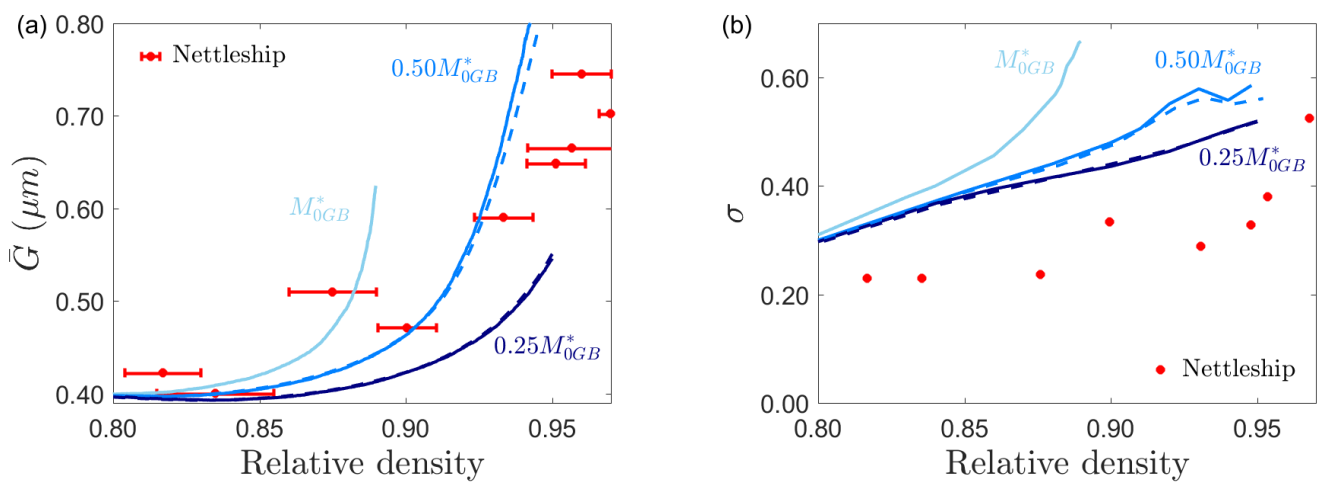

Figure 5: Comparison of DEM simulations with alumina experimental data from Nettleship et al. [66]. Simulations results are obtained using three different values of grainboundary mobility $\left(M_{G B}\right)$ with $M_{0 G B}^{*}$ given in Table 1 . Results for packings made of 40k particles (solid lines) and 400k particles packings (dashed lines) are shown. (a) Mean grain size-density trajectories. (b) $\sigma$ parameter of the lognormal $\left(\mu, \sigma^{2}\right)$ grain size distribution along densification.

Indeed, even if a quantitative evaluation has not been performed, pore coarsening is clearly observed above $D=0.85$. On the last two snapshots, some contact impingements are also noticed. These impingements are in conflict with the DEM hypothesis of independent treatment of the contacts but we believe that, at least in the density range $0.85-0.9$, they are relatively rare events that do not challenge the simulation results. However, above $D=0.9$ the simulation results should be taken with more caution.

\subsubsection{Sintering and grain growth kinetics}

Although Nettleship et al. do not report grain growth kinetics, it is an important output of the simulation that can be investigated in light of the existing classical power laws. Using simulations described above, both relative density and mean grain size are plotted on Fig. 7 as a function of the normalized time $\tau=\frac{r_{m, 0}^{4} k_{b} T}{\gamma_{S} D_{G B} \delta_{G B} \Omega}$. A first qualitative observation is that, in these typical sintering conditions, grain growth slows down densification kinetics. This is a classically observed phenomenon, which is explained both by a decrease of the driving force for sintering and by an increase of the diffusion distances, with the increase of particle size. In addition, simulations show that this decrease in densification rate is pronounced even for a barely perceptible increase in mean grain size and seems to limit the achievable final density. This last point should be taken with care, however, 

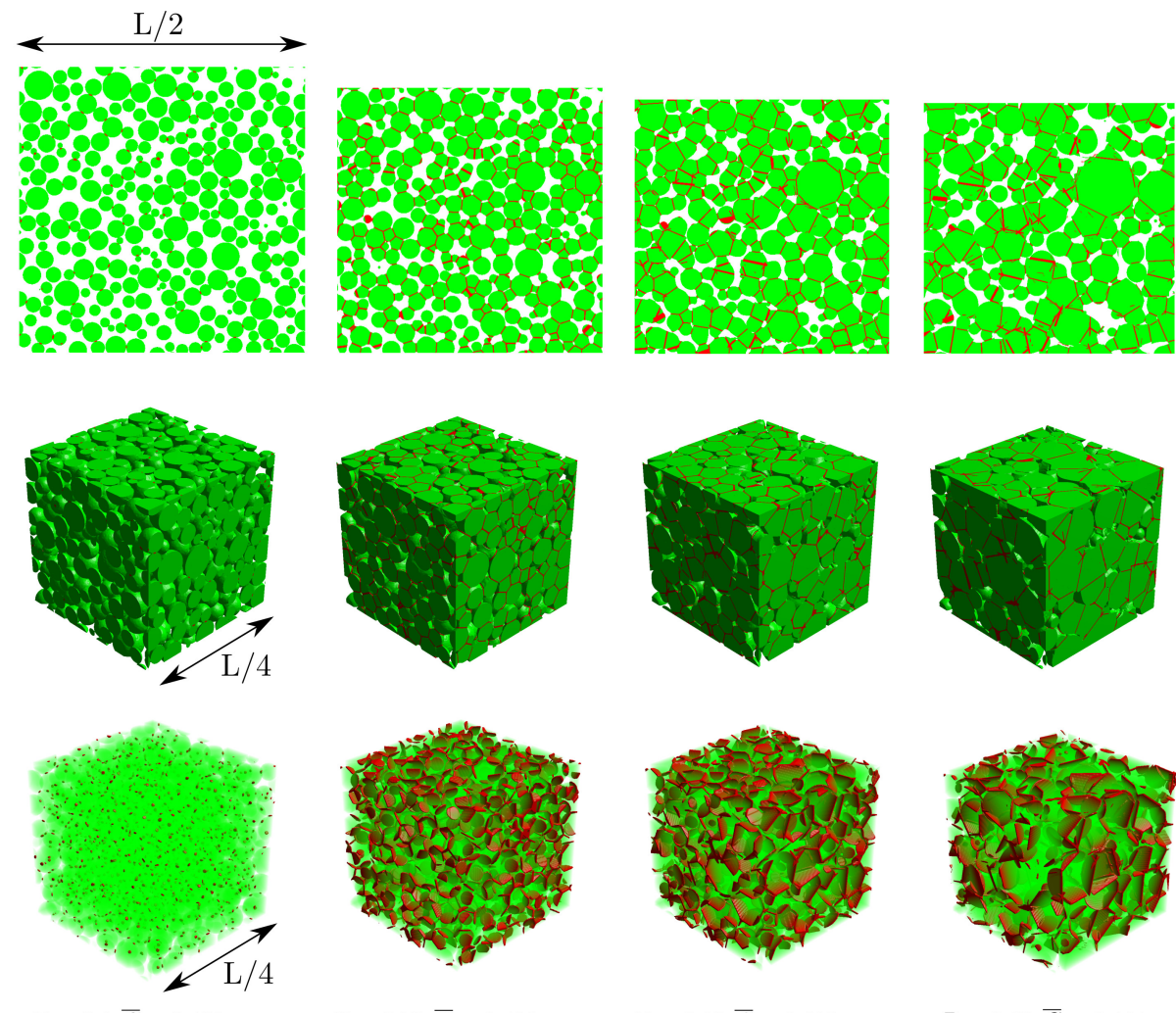

$D=0.6 ; \bar{G}=0.400 \mu m$

$D=0.75 ; \bar{G}=0.400 \mu m$

$D=0.85 ; \bar{G}=0.405 \mu m$

$D=0.89 ; \bar{G}=0.444 \mu m$

Figure 6: Evolution of the microstructure of a $40 \mathrm{k}$ particles packing, $0.5 M_{0 G B}^{*}$ and $\sigma=0.23$. The $2 \mathrm{D}$ and $3 \mathrm{D}$ images have been generated from particles positions and radii, and the computed neck sizes (Eq. (5)). The geometry used for the necks is two inverse tori tangent to each particle (see section S2 of the SI) and GBs are displayed in red. For the sake of clarity only a portion of the total volume $\left(L^{3}\right)$ is shown.

as the DEM intrinsic hypothesis to treat separately the contacts is not met at high densities. An intriguing and also never or rarely experimentally observed point, but reported in the idealized grain growth simulations of Wakai et al. [67], is the observed slight decrease in mean grain size before the expected increase. The explanation is nonetheless very simple: for a given volume transferred from a smaller particle to a larger one, the decrease of the small particle radii will be proportionally more important than the radius change of the larger particles, thus decreasing the mean radius. The subsequent increase in mean particle size is a direct consequence of the decrease of the number of particles, a phenomena only active after some time as observed in Fig. 7b. This time represented by dotted lines represents the 
incubation and transition periods reported by Wakai et al. from which the classical power law $\left(\bar{G}^{n}-\bar{G}_{0}^{n} \propto t\right)$ starts. We obtain $n$ values in between 2.6 and 3.1, which are consistent with experimentally observed values [3,6] but slightly larger than the expected value $n=2$ from theory $[4,8,10]$. This theoretical value has been derived under the assumption of GB migration as the dominant mechanism. The mean field analysis conducted by Hillert [4] or its simplified version proposed by Kang [8] can be applied to Eqs. (9) and (10) (see section S4 of the SI). The theoretical exponents obtained are $n=4$ for grain growth by surface diffusion (Eq. (9)) and, as expected, $n=2$ for grain growth by grain boundary migration (Eq. (10)). It is thus consistent with our results which indicate that $n$ tends to 2 as the grain boundary mobility increases. For the highest value of the grain mobility, abnormal grain growth runaway was triggered in the simulation. This simulation has been stopped at this point as it was not possible to handle it correctly and no power-law fit was attempted. The value $n=4$ predicted by Riedel et al. [68] takes into account the pinning of GB by closed pores which slows down the grain boundary motion. Our model does not take this pinning into account but nevertheless reproduces correctly the experimental data up to quite high densities.

The rate of disappearance of grains is not widely discussed in the solidphase sintering literature. For dense materials [69] or liquid phase sintering [3] it is accepted that the number of grains $N_{p}$ scales with the inverse of time $\left(N_{p} \propto 1 / t\right)$. Fig. 7b indicates that simulations lead to $N_{p} \propto 1 / t^{m}$ with $1.2<m<1.5$, where higher GB mobilities result in higher rates of decay. Note that for longer times $\tau$, the rate of decay slows down and $m$ tends towards values closer to unity.

\subsubsection{Influence of particle size distribution}

Our model can be advantageously used to study the impact of the initial particle size distribution, a task that would be tedious experimentally, and that is hardly accessible by other numerical approaches due to the large number of particles required. It is observed in Fig. 8 that a slight broadening of the initial size distribution can strongly promote early grain growth. This effect of initial particle size distribution is a common experimental observation [3, 70, 71, 72, 73]. From our two particle results in Fig. 4b, we conclude that a direct grain boundary migration (i.e. without surface diffusion stage) is one of the mechanisms for earlier grain growth of wider distributions that necessarily exhibit larger particle size ratios. In addition, the slight initial decrease of the mean grain size (Fig. 8) disappears 

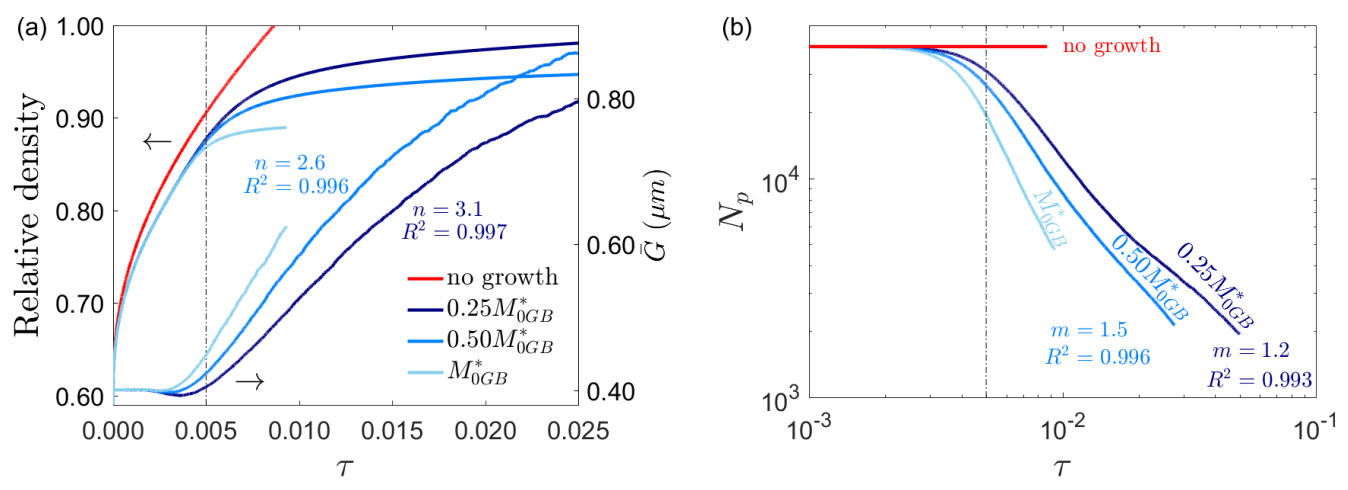

Figure 7: Densification and grain growth kinetics for three values of GB mobility with $M_{0 G B}^{*}$ from Table 1 together with a simulation without any grain growth. Dashed vertical lines indicate the starting time for the fitting curves. (a) Relative density and mean grain size $\bar{G}$ versus normalized time $\tau=\frac{r_{m, 0}^{4} k_{b} T}{\gamma_{S} D_{G B} \delta_{G B} \Omega}$. The power-law exponents $n$ for grain growth $\left(\bar{G}^{n}-\bar{G}_{0}^{n} \propto t\right)$ are indicated together with their R-square values. (b) Number of particles $N_{p}$ (for a 40,000 particles packing) as a function of normalized time $\tau$. The exponent $m$ of the power-law $N_{p} \propto 1 / t^{m}$ is indicated.

for larger size distribution. This explains why this phenomena, although reported in simulations [67], is not observed experimentally.

\subsubsection{Normalized grain size - density trajectory and comparison with ex- perimental data}

The observed large influence of the initial size distribution confirms that any direct comparison with experimental data not reporting it might be doubtful. Still, after the initial assessment of the model using Nettleship data we sought for a broader simulation-experiment comparison. Available data encompasses very different mean (or median) particle sizes, sintering temperatures, purity and size distributions (mostly unknown). Still, Bernard-Granger et al. have demonstrated that $\bar{G}_{0}^{2} / \bar{G}^{2}$ is linear with $D$ [10] under the assumption that the main mechanism for grain growth is GB migration. According to their work, the proportionality coefficient $k$ is a function of $\bar{G}_{0}$ and the ratio of diffusion coefficients $D_{G B M} / D_{G B}$. The temperature has only a minor influence on $k$ through the different activation energies of $D_{G B M}$ and $D_{G B}$, which is consistent with the observation that grain size - density trajectory is not temperature-dependent [10]. Thus, the comparison between our isothermal simulations with experimental data using a heating rate ramp followed by an isothermal dwell is relevant. Sec- 


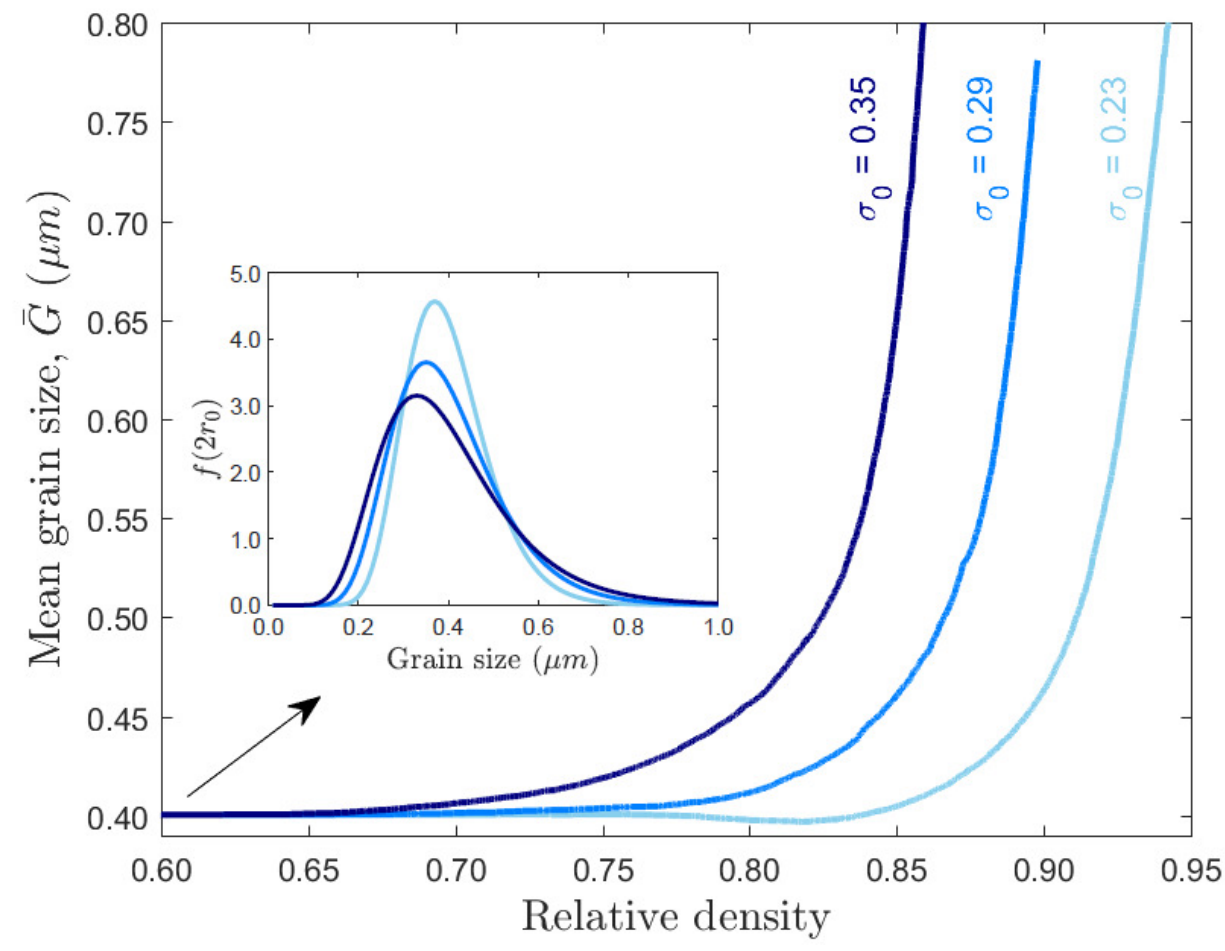

Figure 8: Effect of the initial size distribution on grain growth. Mean grain size $\bar{G}$ as a function of density for three initial standard deviations of the lognormal $\left(\mu_{0}, \sigma_{0}^{2}\right)$ distribution. A wider distribution results in earlier grain growth. The initial size distribution for each sample is shown in the inset.

tion S6 of the SI confirms that the introduction of a realistic heating rate ramp has no significant impact on the grain size - density trajectory. This relationship between grain size and density has actually long been experimentally observed as reported by German [3], but in the form $\bar{G}=\theta \bar{G}_{0} / \epsilon^{1 / 2}$ with $\epsilon=1-D$ the porosity. As illustrated in Fig. 9, plotting $\bar{G}_{0}{ }^{2} / \bar{G}^{2}$ versus relative density is an attractive approach to normalize and report very different experimental data on a single plot. The linearity is clearly confirmed for most of the collected data. German proposed that $\theta$ has generally a value near 0.6. Given that the slope $k$ in Fig. 9 is related to $\theta$ by $\theta=1 /|k|^{1 / 2}$, we obtain a wider range $0.41<\theta<0.72$ from the literature (not considering values from Bae and Baik that are not typical due to the very large particle size). The numerical results also show that $|k|$ decreases with grain size and the values obtained are in agreement with those computed from the theoret- 
ical formula of Bernard-Granger et al. [10], using the same set of parameters (see section S5 of the SI). The simulations bring valuable additional information and show that the decrease of $|k|$ with grain size is associated with an earlier and more progressive, non-linear, onset of grain growth. The influence of a narrower size distribution is a delayed onset of grain growth but without considerable change in $|k|$. A decrease of grain-boundary mobility logically slows grain growth in favor of densification. The onset of grain growth is also slightly delayed to larger densities and the beginning of the trajectory is non-linear with a moderate rate which might be related to more important first stage of grain growth dominated by surface diffusion. Indeed, linearity has been established under the assumption of grain growth by GB migration only. Interestingly, the experiment of Berry et al. $\left(\mathrm{Al}_{2} \mathrm{O}_{3}\right.$ without $\mathrm{MgO}$ doping $)$ also exhibits a non-linear trajectory but with a different shape as compared to the simulations. The same curve behavior of Berry et al. is observed in Greskovich and Lay [74] and in Zhao and Harmer [63]. While the simulation trajectory has a convex shape (increasing grain growth - density rate) the experiment trajectory has a concave shape (decreasing grain-growth density rate). This latter behavior might be explained by a decreasing GB mobility at the onset of pore closure due to an associated increase of pore drag. In addition, pore and grain sizes could also influence the GB mobility through varying amount of drag effects. Introducing a density or grain size dependant grain boundary mobility could thus make some sense and help to model more correctly some experimental cases.

It can be concluded that, by adjusting the particle size distribution and GB mobility, the model has the ability to reproduce the large range of observed grain growth - density trajectories. However, we believe that using these as two fitting parameters might not be relevant since the involved intercorrelated phenomena might be too complex to be caught by a simple variation of grain size with density.

\section{Conclusion}

Sintering and grain growth are a highly coupled phenomena with shrinkage, surface diffusion, grain-boundary migration and particle coalescence arising simultaneously, that poses a challenge to current simulation methods. Until now, these couplings have only been successfully treated by mesoscale phase-field or Monte Carlo methods. By taking full advantage of the 3D discontinuous discrete element framework, simulations presented 

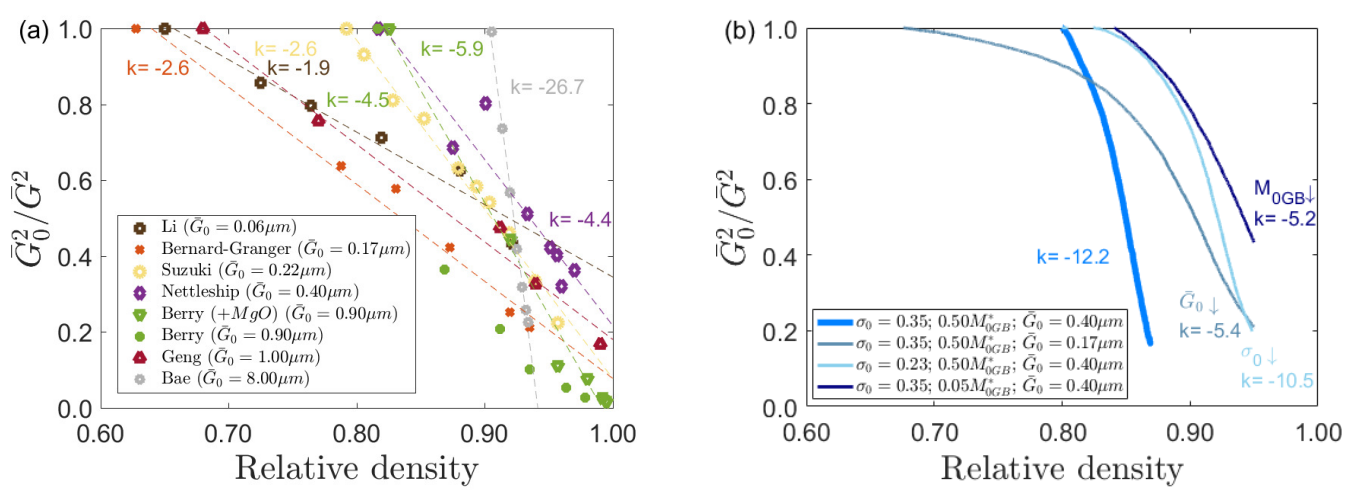

Figure 9: Grain size-density trajectories. (a) Experimental data (symbols) from Li and Ye [75], Bernard-Granger and Guizard [9], Geng et al. [76], Nettleship et al. [66], Suzuki et al. [77], Berry and Harmer [11] and Bae and Baik [12]. Dashed lines show linear fits with the corresponding slope $k$. (b) Simulations results obtained for different initial mean grain size $\bar{G}_{0}$, initial grain size distribution $\sigma_{0}$ and grain-boundary mobility $M_{0 G B}$.

here provide an alternative that has the ability to treat very large systems. The adopted model treats nonetheless the main fluxes of matter between particles through physically-based interaction laws to provide reasonable accuracy. Its limitation lies mainly on the assumption that interactions between particles are handled as pairs. As densification progresses, contact impingement becomes more likely which restricts the domain of quantitative validity of our DEM simulations to initial and intermediate sintering stages $(D \leq 0.90-0.95)$. Also, our model considers a grain-boundary mobility that only depends on temperature. This is questionable for the final stage of sintering for which the pore drag force on grain-boundary mobility can be significant. Still, for initial and intermediate stages, using reasonable material parameters from the literature the model correctly reproduce experimental mean grain size evolution for alumina. For realistic particle size distribution, grain growth can affect the sintering kinetics and the microstructure evolution early in the first stage and in the intermediate stage of sintering. Hence, the realism of DEM simulation of sintering is largely improved by the present model as compared to earlier DEM approaches that do not account for grain growth. Taking advantage of the large systems tractable by DEM, the influence of initial particle size distribution on grain growth has been studied. The DEM simulations show, as reported from experiments [3], that broader particle size distributions exhibit faster grain growth. This points to some potential avenues for retarding grain 
growth with narrower initial size distributions. However, narrow size distributions are difficult to achieve experimentally and lead to a smaller green density that is detrimental for densification. The present DEM model might help to optimally choose the size distribution for a given system. As largescale simulations are feasible with DEM (up to 400000 particles have been treated here), future work will address more complex sintering conditions (sintering on a substrate, composites, presence of defects, stress-assisted sintering, two-step sintering...). Improvements of the model should for example consider the use of a porosity or impurity dependent GB mobility. This should provide a better understanding of the conditions, strongly dependent on GB mobility, that lead either to the emergence of a self-similar grain size distribution or, on the contrary, to abnormal grain growth. Further improvements of the model, however, will be hampered by a lack of experimental data for its reliable assessment.

\section{Acknowledgements}

This project has received funding from the European Union's Horizon 2020 research and innovation programme under the Marie Skłodowska-Curie grant agreement MATHEGRAM No 813202. Some of the computations presented in this paper were performed using the GRICAD infrastructure (https://gricad.univ-grenoble-alpes.fr), which is supported by Grenoble research communities.

\section{Supplementary data}

Supplementary information and video associated with this article can be found in the online version.

\section{References}

[1] R. M. German, Sintering: from Empirical Observations to Scientific Principles, Butterworth-Heinemann, Boston, 2014.

[2] F. F. Lange, B. J. Kellett, Thermodynamics of Densification: II, Grain Growth in Porous Compacts and Relation to Densification, Journal of the American Ceramic Society 72 (5) (1989) 735-741. doi:10.1111/j.1151-2916.1989.tb06209.x.

[3] R. M. German, Coarsening in sintering: Grain shape distribution, grain size distribution, and grain growth kinetics in solid-pore systems, Critical Reviews in Solid State and Materials Sciences 35 (4) (2010) 263-305. doi:10.1080/10408436.2010.525197. 
[4] M. Hillert, On the theory of normal and abnormal grain growth, Acta Metallurgica 13 (3) (1965) 227-238. doi:10.1016/0001-6160(65)90200-2.

[5] M. Rahaman, Ceramic processing and sintering, second edition, CRC Press, 2003. doi:10.1201/9781315274126.

[6] W. D. Kingery, B. François, Grain Growth in Porous Compacts, Journal of the American Ceramic Society 48 (10) (1965) 546-547. doi:10.1111/j.11512916.1965.tb14665.x.

[7] R. M. German, The contiguity of liquid phase sintered microstructures, Metallurgical Transactions A 16 (7) (1985) 1247-1252. doi:10.1007/BF02670329.

[8] S.-J. L. Kang, Sintering Densification, Grain Growth, and Microstructure, Butterworth-Heinemann, Oxford, 2005. doi:https://doi.org/10.1016/B978075066385-4/50000-5.

[9] G. Bernard-Granger, C. Guizard, New relationships between relative density and grain size during solid-state sintering of ceramic powders, Acta Materialia 56 (20) (2008) 6273-6282. doi:10.1016/j.actamat.2008.08.054.

[10] G. Bernard-Granger, N. Monchalin, C. Guizard, Sintering of ceramic powders: Determination of the densification and grain growth mechanisms from the "grain size/relative density" trajectory, Scripta Materialia 57 (2) (2007) 137-140. doi:10.1016/j.scriptamat.2007.03.030.

[11] K. A. Berry, M. P. Harmer, Effect of MgO Solute on Microstructure Development in Al2O3, Journal of the American Ceramic Society 69 (2) (1986) 143-149. doi:10.1111/j.1151-2916.1986.tb04719.x.

[12] I.-J. Bae, S. Baik, Abnormal Grain Growth of Alumina, Journal of the American Ceramic Society 80 (5) (1997) 1149-1156. doi:10.1111/j.1151-2916.1997.tb02957.x.

[13] J. Pan, H. Le, S. Kucherenko, J. A. Yeomans, A model for the sintering of spherical particles of different sizes by solid state diffusion, Acta Materialia 46 (13) (1998) 4671-4690. doi:10.1016/S1359-6454(98)00144-X.

[14] D. Zhang, A. Weng, S. Gong, D. Zhou, Computer simulation of grain growth of intermediate* / and final-stage sintering and Ostwald ripening of $\mathrm{BaTiO}$ 3based PTCR ceramics, Materials Science and Engineering: B 99 (2003) 428-432. doi:10.1016/S0921-5107(02)00449-X.

[15] H. Itahara, T. Tani, H. Nomura, H. Matsubara, Computational design for grain-oriented microstructure of functional ceramics prepared by templated grain growth, Journal of the American Ceramic Society 89 (5) (2006) 1557-1562. doi:10.1111/j.1551-2916.2006.00954.x.

[16] V. Tikare, M. Braginsky, D. Bouvard, A. Vagnon, Numerical simulation of microstructural evolution during sintering at the mesoscale in a 3D powder compact, Computational Materials Science 48 (2) (2010) 317-325. doi:10.1016/j.commatsci.2010.01.013.

[17] S. Hara, A. Ohi, N. Shikazono, Sintering analysis of sub-micron-sized nickel powders: Kinetic Monte Carlo simulation verified by FIB-SEM reconstruction, Journal of Power Sources 276 (2015) 105-112. doi:10.1016/j.jpowsour.2014.11.110.

[18] Y. Zhang, X. Xiao, J. Zhang, Kinetic Monte Carlo simulation of sintering behavior of additively manufactured stainless steel powder particles using reconstructed microstructures from synchrotron X-ray microtomography, Results in Physics 13 (jun 2019). doi:10.1016/j.rinp.2019.102336. 
[19] A. Kazaryan, Y. Wang, S. A. Dregia, B. R. Patton, Grain growth in systems with anisotropic boundary mobility: Analytical model and computer simulation, Physical Review B - Condensed Matter and Materials Physics 63 (18) (2001) 1-11. doi:10.1103/PhysRevB.63.184102.

[20] K. Asp, J. Ågren, Phase-field simulation of sintering and related phenomena - A vacancy diffusion approach, Acta Materialia 54 (5) (2006) 1241-1248. doi:10.1016/j.actamat.2005.11.005.

[21] Y. U. Wang, Computer modeling and simulation of solid-state sintering: A phase field approach, Acta Materialia 54 (4) (2006) 953-961. doi:10.1016/j.actamat.2005.10.032.

[22] V. Kumar, Z. Z. Fang, P. C. Fife, Phase field simulations of grain growth during sintering of two unequal-sized particles, Materials Science and Engineering A 528 (1) (2010) 254-259. doi:10.1016/j.msea.2010.08.061.

[23] K. Ahmed, C. A. Yablinsky, A. Schulte, T. Allen, A. El-Azab, Phase field modeling of the effect of porosity on grain growth kinetics in polycrystalline ceramics, Modelling and Simulation in Materials Science and Engineering 21 (6) (2013). doi:10.1088/0965-0393/21/6/065005.

[24] S. Biswas, D. Schwen, J. Singh, V. Tomar, A study of the evolution of microstructure and consolidation kinetics during sintering using a phase field modeling based approach, Extreme Mechanics Letters 7 (2016) 78-89. doi:10.1016/j.eml.2016.02.017.

[25] F. Wakai, M. Yoshida, Y. Shinoda, T. Akatsu, Coarsening and grain growth in sintering of two particles of different sizes, Acta Materialia 53 (5) (2005) 1361-1371. doi:10.1016/j.actamat.2004.11.029.

[26] J. Bruchon, D. Pino-Muñoz, F. Valdivieso, S. Drapier, Finite element simulation of mass transport during sintering of a granular packing. Part I. Surface and lattice diffusions, Journal of the American Ceramic Society 95 (8) (2012) 2398-2405. doi:10.1111/j.1551-2916.2012.05073.x.

[27] C. L. Martin, L. C. Schneider, L. Olmos, D. Bouvard, Discrete element modeling of metallic powder sintering, Scripta Materialia 55 (5) (2006) 425-428. doi:10.1016/j.scriptamat.2006.05.017.

[28] K. Shinagawa, Simulation of grain growth and sintering process by combined phase-field/discrete-element method, Acta Materialia 66 (2014) 360-369. doi:10.1016/j.actamat.2013.11.023.

[29] T. Matsuda, Development of a DEM taking account of neck increments caused by surface diffusion for sintering and application to analysis of the initial stage of sintering, Computational Materials Science 196 (February) (2021) 110525. doi:10.1016/j.commatsci.2021.110525.

[30] M. W. Reiterer, K. G. Ewsuk, An analysis of four different approaches to predict and control sintering, Journal of the American Ceramic Society 92 (7) (2009) 1419-1427. doi:10.1111/j.1551-2916.2009.03009.x.

[31] V. Rehn, J. Hötzer, W. Rheinheimer, M. Seiz, C. Serr, B. Nestler, Phase-field study of grain growth in porous polycrystals, Acta Materialia 174 (2019) 439-449. doi:10.1016/j.actamat.2019.05.059.

[32] J. Hötzer, M. Seiz, M. Kellner, W. Rheinheimer, B. Nestler, Phase-field simulation of solid state sintering, Acta Materialia 164 (2019) 184-195. doi:10.1016/j.actamat.2018.10.021. 
[33] R. Termuhlen, X. Chatzistavrou, J. D. Nicholas, H. C. Yu, Three-dimensional phase field sintering simulations accounting for the rigid-body motion of individual grains, Computational Materials Science 186 (June 2020) (2021) 109963. doi:10.1016/j.commatsci.2020.109963.

[34] F. Parhami, R. M. McMeeking, A network model for initial stage sintering, Mechanics of Materials 27 (2) (1998) 111-124. doi:10.1016/S0167-6636(97)00034-3.

[35] P. Cundall, O. Strack, A discrete numerical model for granular assemblies, Géotechnique (1979) 47-65doi:10.1016/0148-9062(79)91211-7.

[36] B. Henrich, A. Wonisch, T. Kraft, M. Moseler, H. Riedel, Simulations of the influence of rearrangement during sintering, Acta Materialia 55 (2) (2007) 753-762. doi:10.1016/j.actamat.2006.09.005.

[37] C. L. Martin, R. K. Bordia, The effect of a substrate on the sintering of constrained films, Acta Materialia 57 (2) (2009) 549-558. doi:10.1016/j.actamat.2008.09.041.

[38] C. Wang, S. Chen, Application of the complex network method in solid-state sintering, Computational Materials Science 69 (2013) 14 - 21 . doi:https://doi.org/10.1016/j.commatsci.2012.11.020.

[39] S. Martin, M. Guessasma, J. Léchelle, J. Fortin, K. Saleh, F. Adenot, Simulation of sintering using a Non Smooth Discrete Element Method. Application to the study of rearrangement, Computational Materials Science 84 (2014) 31-39. doi:10.1016/j.commatsci.2013.11.050.

[40] R. Besler, M. Rossetti Da Silva, J. J. Do Rosario, M. Dosta, S. Heinrich, R. Janssen, Sintering Simulation of Periodic Macro Porous Alumina, Journal of the American Ceramic Society 98 (11) (2015) 3496-3502. doi:10.1111/jace.13684.

[41] S. Nosewicz, J. Rojek, M. Chmielewski, Discrete Element Framework for Determination of Sintering and Postsintering Residual Stresses of, Materials 13 (4015) (2020) 1-21. doi:10.3390/ma13184015.

[42] A. Wonisch, T. Kraft, M. Moseler, H. Riedel, Effect of different particle size distributions on solid-state sintering: A microscopic simulation approach, Journal of the American Ceramic Society 92 (7) (2009) 1428-1434. doi:10.1111/j.15512916.2009.03012.x.

[43] S. Martin, S. Navarro, H. Palancher, A. Bonnin, J. Léchelle, M. Guessasma, J. Fortin, K. Saleh, Validation of DEM modeling of sintering using an in situ X-ray microtomography analysis of the sintering of $\mathrm{NaCl}$ powder, Computational Particle Mechanics 3 (4) (2016) 525-532. doi:10.1007/s40571-015-0062-7.

[44] L. Olmos, C. L. Martin, D. Bouvard, D. Bellet, M. Di Michiel, Investigation of the sintering of heterogeneous powder systems by synchrotron microtomography and discrete element simulation, Journal of the American Ceramic Society 92 (7) (2009) 1492-1499. doi:10.1111/j.1551-2916.2009.03037.x.

[45] D. Bouvard, R. M. McMeeking, Deformation of Interparticle Necks by DiffusionControlled Creep (1996). doi:10.1111/j.1151-2916.1996.tb07927.x.

[46] C. L. Martin, H. Camacho-Montes, L. Olmos, D. Bouvard, R. K. Bordia, Evolution of defects during sintering: Discrete element simulations, Journal of the American Ceramic Society 92 (7) (2009) 1435-1441. doi:10.1111/j.1551-2916.2009.03014.x.

[47] Z. Yan, C. L. Martin, O. Guillon, D. Bouvard, C. S. Lee, Microstructure evolution during the co-sintering of $\mathrm{Ni} / \mathrm{BaTiO} 3$ multilayer ceramic capacitors modeled by discrete element simulations, Journal of the European Ceramic Society 34 (13) (2014) 
3167-3179. doi:10.1016/j.jeurceramsoc.2014.04.013.

[48] J. R. Carazzone, C. L. Martin, Z. C. Cordero, Crack initiation, propagation, and arrest in sintering powder aggregates, Journal of the American Ceramic Society (2020) 1-20doi:10.1111/jace.17170.

[49] V. Ogarko, S. Luding, A fast multilevel algorithm for contact detection of arbitrarily polydisperse objects, Computer Physics Communications 183 (4) (2012) 931-936. doi:10.1016/j.cpc.2011.12.019.

[50] J. Christoffersen, M. M. Mehrabadi, S. Nemat-Nasser, A micromechanical description of granular material behavior, Journal of Applied Mechanics 48 (1981) 339-344.

[51] F. Parhami, R. M. McMeeking, A. C. Cocks, Z. Suo, A model for the sintering and coarsening of rows of spherical particles, Mechanics of Materials 31 (1) (1999) 43-61. doi:10.1016/S0167-6636(98)00049-0.

[52] R. L. Coble, Initial Sintering of Alumina and Hematite, Journal of the American Ceramic Society 41 (2) (1958) 55-62.

[53] C. Herring, Diffusional viscosity of a polycrystalline solid, Journal of Applied Physics 21 (5) (1950) 437-445. doi:10.1063/1.1699681.

[54] S. J. L. Kang, Y. I. Jung, Sintering kinetics at final stage sintering: Model calculation and map construction, Acta Materialia 52 (15) (2004) 4573-4578. doi:10.1016/j.actamat.2004.06.015.

[55] F. Humphreys, A unified theory of recovery, recrystallization and grain growth, based on the stability and growth of cellular microstructures-I. The basic model, Acta Materialia 45 (10) (1997) 4231-4240. doi:10.1016/S1359-6454(97)00070-0.

[56] S. J. Dillon, M. P. Harmer, Intrinsic grain boundary mobility in alumina, Journal of the American Ceramic Society 89 (12) (2006) 3885-3887. doi:10.1111/j.15512916.2006.01331.x.

[57] R. J. BROOK, Pore-Grain Boundary Interactions and Grain Growth, Journal of the American Ceramic Society 52 (1) (1969) 56-57. doi:10.1111/j.11512916.1969.tb12664.x.

[58] J. W. Rutter, K. T. Aust, Migration of 100 tilt grain boundaries in high purity lead, Acta Metallurgica 13 (3) (1965) 181-186. doi:10.1016/0001-6160(65)90194-X.

[59] G. Gottstein, D. A. Molodov, L. S. Shvindlerman, Grain boundary migration in metals: recent developments, Interface Science 6 (1-2) (1998) 7-22. doi:10.1023/a:1008641617937.

[60] J. E. Burke, D. Turnbull, Recrystallization and grain growth, Progress in Metal Physics 3 (C) (1952). doi:10.1016/0502-8205(52)90009-9.

[61] A. Tsoga, P. Nikolopoulos, Groove Angles and Surface Mass Transport in Polycrystalline Alumina, Journal of the American Ceramic Society 77 (4) (1994) 954-960. doi:10.1111/j.1151-2916.1994.tb07252.x.

[62] W. M. Robertson, R. Chang, The kinetics of grain-boundary groove growth on alumina surfaces, in: W. W. Kriegel, H. Palmour (Eds.), The Role of Grain Boundaries and Surfaces in Ceramics, Springer US, Boston, MA, 1966, pp. 49-60.

[63] J. Zhao, M. P. Harmer, Sintering of Ultra-High-Purity Alumina Doped Simultaneously with $\mathrm{MgO}$ and FeO, Journal of the American Ceramic Society 70 (12) (1987) 860-866. doi:10.1111/j.1151-2916.1987.tb04906.x.

[64] A. Wonisch, O. Guillon, T. Kraft, M. Moseler, H. Riedel, J. Rödel, Stress-induced anisotropy of sintering alumina: Discrete element modelling and experiments, Acta 
Materialia 55 (15) (2007) 5187-5199. doi:10.1016/j.actamat.2007.05.038.

[65] O. A. Ruano, J. Wadsworth, O. D. Sherby, Deformation of fine-grained alumina by grain boundary sliding accommodated by slip, Acta Materialia 51 (12) (2003) 3617-3634. doi:10.1016/S1359-6454(03)00180-0.

[66] I. Nettleship, R. J. McAfee, W. S. Slaughter, Evolution of the grain size distribution during the sintering of alumina at $1350^{\circ} \mathrm{c}$, Journal of the American Ceramic Society 85 (8) (2002) 1954-1960. doi:10.1111/j.1151-2916.2002.tb00387.x.

[67] F. Wakai, N. Enomoto, H. Ogawa, Three-dimensional microstructural evolution in ideal grain growth general statistics, Acta Materialia 48 (6) (2000) 1297-1311. doi:10.1016/S1359-6454(99)00405-X.

[68] H. Riedel, J. Svoboda, A theoretical study of grain growth in porous solids during sintering, Acta Metallurgica Et Materialia 41 (6) (1993) 1929-1936. doi:10.1016/0956-7151(93)90212-B.

[69] F. N. Rhines, K. R. Craig, R. T. DeHoff, Mechanism of steady-state grain growth in aluminum, Metallurgical Transactions 5 (2) (1974) 413-425. doi:10.1007/BF02644109.

[70] B. R. Patterson, V. D. Parkhe, Particle Size Distribution Effects on Sintering of Spherical Tungsten., Progress in Powder Metallurgy 41 (August) (1986) 347-354.

[71] Z. Fang, B. R. Patterson, Experimental investigation of particle size distribution influence on diffusion controlled coarsening, Acta Metallurgica Et Materialia 41 (7) (1993) 2017-2024. doi:10.1016/0956-7151(93)90372-Y.

[72] J. M. Ting, R. Y. Lin, Effect of particle size distribution on sintering - Part II Sintering of alumina, Journal of Materials Science 30 (9) (1995) 2382-2389. doi:10.1007/BF01184590.

[73] R. Bjørk, V. Tikare, H. L. Frandsen, N. Pryds, The effect of particle size distributions on the microstructural evolution during sintering, Journal of the American Ceramic Society 96 (1) (2013) 103-110. doi:10.1111/jace.12100.

[74] C. Greskovich, K. W. Lay, Grain Growth in Very Porous Al2O3 Compacts, Journal of the American Ceramic Society 55 (3) (1972) 142-146. doi:10.1111/j.11512916.1972.tb11238.x.

[75] J. Li, Y. Ye, Densification and grain growth of Al2O3 nanoceramics during pressureless sintering, Journal of the American Ceramic Society 89 (1) (2006) 139-143. doi:10.1111/j.1551-2916.2005.00654.x.

[76] X. Geng, Y. Hong, J. Lei, J. Ma, J. Chen, H. Xiao, J. Tong, R. K. Bordia, F. Peng, Ultra-fast, selective, non-melting, laser sintering of alumina with anisotropic and size-suppressed grains, Journal of the American Ceramic Society 104 (5) (2021) 1997-2006. doi:10.1111/jace.17617.

[77] M. Suzuki, K. Kawazoe, Effective surface diffusion coefficients of volatile organics on activated carbon during adsorption from aqueous solution, Journal of Chemical Engineering of Japan 8 (5) (1975) 379-382. doi:10.1252/jcej.8.379. 